\section{Determining the origins of particulates on Arkaroo Rock art}

\author{
Kate Colyer, ${ }^{1,2}$ Jamie S. Quinton, ${ }^{1}$ \\ Allan Pring, ${ }^{2}$ Rachel S. Popelka-Filcoff, \\ Claire E. Lenehan'
}

'School of Chemical and Physical Sciences, Flinders University, Adelaide;

2Department of Mineralogy, South

Australian Museum, Adelaide, Australia

\section{Abstract}

Arkaroo Rock, an important Aboriginal art site located in the Flinders Ranges National Park, South Australia, features a range of artworks created using red and yellow ochre along with white minerals (such as kaolinite, and gypsum) and charcoal. The site is widely promoted to tourists and, during peak season, attracts more than fifty visitors each day. In mid 2007, the local indigenous community noted that the art was covered with a dust-like substance, the nature of which was unknown. It was hypothesised that the substance was the result of local soil adhering to the surface of the artwork with the morning dew. In order to preserve the site, cleaning and conservation work began in early 2008. This study presents an investigation into the source of the particulate matter covering the artworks. Prior to the conservation work commencing, samples were collected from various locations on the rock, within the protective fence surrounding the artwork, and the surrounding soil. Plant samples were also collected proximal to the artwork. A number of techniques were used to characterise and identify the origin of the particulate material from the rock surface. These techniques included optical microscopy, X-ray diffraction (XRD), thermal gravimetric analysis (TGA), colour analysis based on the ES-1000 EFI spectrophotometer (UV-Vis), and subsequent multivariate statistical analysis. Results of this study are presented here, including detailed results obtained from an emerging colour-based ES-1000 EFI spectrophotometer method along with TGA, optical microscopy and XRD. Results confirm the presence of morning dew in surface samples, and eliminate local sources as the origin of the particulate matter.

\section{Introduction}

Ochre is an extremely common, naturally occurring pigment ranging in colour from yellow to deep purple with the primary chro- mophore being an iron-oxide (Bell et al., 1997). In general, red-coloured ochres contain haematite $\left(\alpha-\mathrm{Fe}_{2} \mathrm{O}_{3}\right)$ as the predominant pigment component, while yellow ochres typically contain goethite $\left(\alpha-\mathrm{FeOOH}\right.$ or $\left.\mathrm{Fe}_{2} \mathrm{O}_{3} \cdot \mathrm{H}_{2} \mathrm{O}\right)$ as the predominant colour component (Elias et al., 2006; Marshall et al., 2005).

The use of these naturally-occurring ochre pigments is widespread amongst all Aboriginal Australian groups and continues from the earliest documented human occupation of Australia until today (Sagona, 1994). Ochre is of particular significance and many archaeological finds confirm both its use and its significance to the Aboriginal culture (Sagona, 1994; Jercher, 1998). Because of its high colouring capacity as well as proven stability under a variety of weather conditions, ochre has been used in a variety of contexts for cultural and ritualistic purposes (Bikiaris, 2000). These include its use as a pigment in body decoration and on a variety of culturally important materials including cave walls, rock shelters and wooden objects. Whilst these pigments are relatively inert and stable, rock art is susceptible to damage from a variety of sources, both natural and anthropogenic. These include subsidence (rock fall), vandalism, weathering and exposure to transported aerosols and airborne dusts.

It has been reported that dusts, whether airborne or from trampling of the floor sediment, can be a contributing factor in the deterioration of rock art, and accumulates in sheltered locations (Watchman and Jones, 1998; Morwood, 1994). This study focuses on the characterisation of dust-like particulate matter found coating rock paintings at Arkaroo Rock, a site within the Flinders Ranges National Park, located approximately $450 \mathrm{~km}$ north of the Adelaide in South Australia (Figure 1).

Arkaroo Rock is an Adnyamathanha (meaning hills people) art site named for Akurra, the Dreaming serpent responsible for the creation of the Flinders Ranges, and more specifically, Wilpena Pound. The ochre and charcoal paintings present at the site depict this Dreaming story (Klaassen, 2007). The site is heavily promoted to tourists and has undergone extensive remodelling since the mid 1980 s, including the erection of a cage/protective fence and boardwalk surrounding the art in an attempt to protect it from its thousands of annual visitors (Klaassen, 2007; Birt, 2007).

In mid-2007, dust-like particles covering the surface of the artwork at Arkaroo Rock were identified by Mr Ralph Coulthard, a local Adnyamathanha man and employee of the Aboriginal Partnerships Section, Department for Environment and Heritage (DEH). Photographs taken at this time clearly show the presence of the particulate matter (Figure 2). This has been detected previously and conservation work carried out at the site (1980-1988) included the construction of a caged boardwalk
Correspondence: Claire Lenehan, School of Chemical and Physical Sciences, Flinders University, Sturt Road, Adelaide, Australia.

Tel.Fax: +61.8.82012191.

E-mail: claire.lenehan@flinders.edu.au

Key words: aboriginal Australian archaeology, spectroscopic methods, ochre, Arkaroo Rock.

Acknowledgements: the authors wish to thank $\mathrm{Mr}$ Peter Birt, Acting Senior Heritage Officer, and Ms Penny Karamanlis, Senior Heritage Officer, Aboriginal Affairs and Reconciliation Division from The Department of the Premier and Cabinet; Mr Dave Lambert, a conservator with the New South Wales National Parks and Wildlife Service; Mr Ralph Coulthard, a local Adnyamathanha man and employee of the Aboriginal Partnerships Section, Department for Environment and Heritage (DEH), Indigenous Engagement Officer, South Australian Arid Lands NRM Board; Mr Tom McIntosh, Senior Ranger, and Mr Arthur Coulthard, Adnymathanha Ranger Department for Environment and Heritage; Ms Pauline Coulthard, local Aboriginal heritage committee member; and Mr Cliff Coulthard, Native title claimant, and all other involved, for allowing us to be a part of this project.

Citation: Colyer K, Quinton JS, Pring A, PopelkaFilcoff RS, Lenehan CE, 2014. Determining the origins of particulates on Arkaroo Rock art. In: $\mathrm{RH}$ Tykot (ed.), Proceedings of the $38^{\text {th }}$ International Symposium on Archaeometry - May $10^{\text {th }}-14^{\text {th }}$ 2010, Tampa, Florida. Open Journal of Archaeometry 2:5301.

Presented at the $38^{\text {th }}$ International Symposium on Archaeometry - May $10^{\text {th }}-14^{\text {th }} 2010$, Tampa, Florida.

This work is licensed under a Creative Commons Attribution 3.0 License (by-nc 3.0).

(C) Copyright K. Colyer et al., 2014

Licensee PAGEPress, Italy

Open Journal of Archaeometry 2014; 2:5301

doi:10.4081/arc.2014.5301

to prevent disturbance of the local flora and ground. However, despite these precautions, the local community has noticed the presence of particulate matter on the rock paintings has increased with time.

Samples were collected and analysis undertaken in an attempt to identify the particulate and its origin, and to confirm the hypothesis that the fine particles collecting on the surface are being blown into the area with the breeze and are adhering to the surface of the rock when dew is present, and are not a result of soil disturbance in and around the caged area.

During conservation work in April 2008, samples were collected from the areas shown in Figure 2. Samples 1-6 and 12 are of the dustlike particles collected from the surface of the rock art and were collected with an artist's 
brush and sterile glass containers, collecting as much sample as was obtainable from within an area of approximately $5 \mathrm{~cm}^{2}$ (approximately 2 g). Samples 3-4 were collected as they were from an area on the rock art showing suspected salt leaching. Samples 7-10 are also approximately $2 \mathrm{~g}$ in weight, and are composed of soil collected from within the protective fence and surrounding area, and sample 11 is various plant material, including small leaves and stems, collected from a plant growing within the protective fence.

\section{Materials and Methods}

Optical microscopy was performed using a Leitz orthopol 12 petrographic microscope (Leica, Wetzlar, Germany), with magnification between 50 and 200 times. Samples were prepared with approximately 0.3 grams of sample in 1-2 drops of Canadian Balsam and analysis was performed at the South Australian Museum.

X-ray diffraction (XRD) was performed on samples approximately 0.0025 grams in weight,

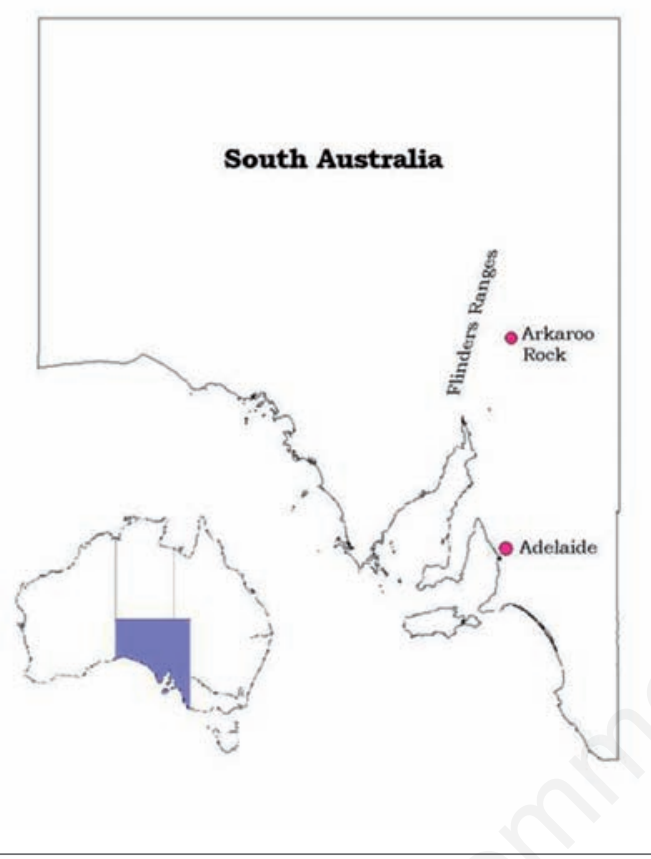

Figure 1. The location of Arkaroo Rock relative to South Australia's capital city, Adelaide.

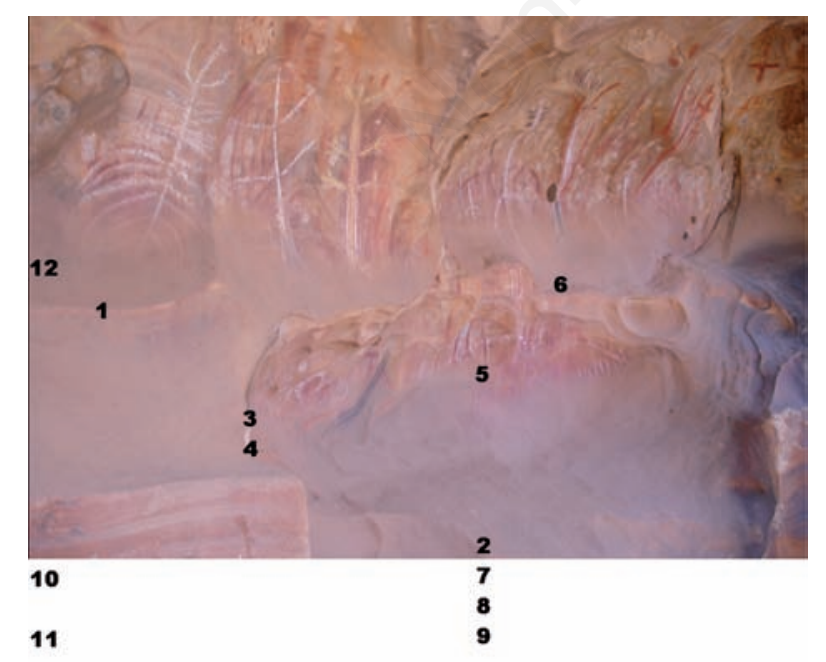

Figure 2. Photograph showing the dust-like particles collecting on the surface of the artwork, and the areas where samples were collected both from the rock surface (samples 1-6, 12) and from ground within the protective fence (samples 7-11). ground in a mortar and pestle, then adhered to Mylar film with acetone following the addition of approximately $10 \%$ silicon powder as a phase standard with an exposure time of 120 minutes. Powder X-ray diffraction patterns were collected on a Huber Guinier Imaging Plate G670 with Co $\mathrm{K}_{\alpha 1}$ radiation $(\lambda=1.78892 \AA)$ generated at $35 \mathrm{kV}$ and $34 \mathrm{~mA}$, and processed using Jade software. Quantitative phase analyses were performed using the Rietveld method with the aid of a computer program Rietica for Windows (v1.7.7).

Thermal gravimetric analysis (TGA) was performed on an Auto TGA 2950HR version 6.1A. Samples were ground but otherwise un-treated, and sample size is in the range of tens of milligrams. Samples were analysed under compressed air and heated from room temperature (approximately $25^{\circ} \mathrm{C}$ ) to $900^{\circ} \mathrm{C}$ at a rate of approximately $10^{\circ} \mathrm{C}$ per minute. The weight is constantly monitored and is given as a function of temperature.

Colour measurements were taken using an

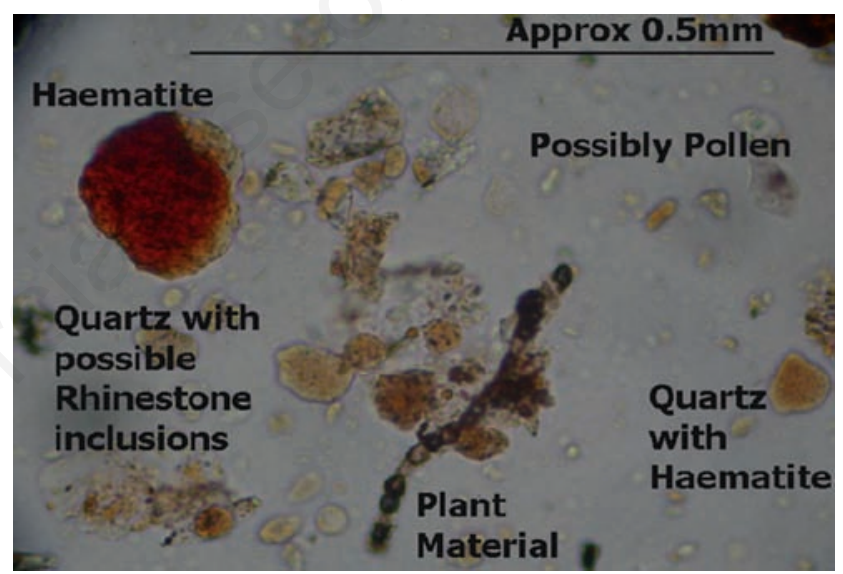

Figure 3. Photograph taken on medium magnification utilising a petrographic microscope showing a number of the species present in sample number 5 of the dust-like particulate removed from the rock surface.

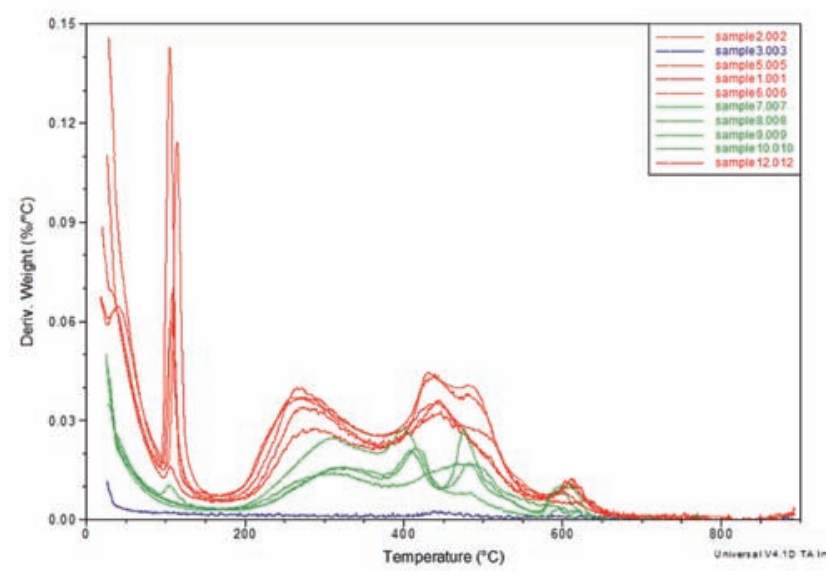

Figure 4. Results of the thermal gravimetric analysis of the fine particles collected from Arkaroo Rock. The plot shows the derivative weight (percent) as a function of temperature. 
EFI-ES1000 hand-held spectrophotometer (Electronics For Imaging Inc., Fremont, CA, USA) capable of performing measurements in the region of $380-730 \mathrm{~nm}$. The instrument has a gas-filled tungsten type a light source, combined with an LED array, UV-filter, and a holographic diffraction grating analyser with 128 pixel diode array. The measurement geometry is from $45^{\circ} / 0^{\circ}$ from a dual channel, and spectral data is collected at $10 \mathrm{~nm}$ steps in reflectance mode. The illumination diameter is $7 \mathrm{~mm}$ with a measurement aperture of 4-4.5 mm diameter. Output is in the forms of XYZ, CIE, $\mathrm{L}^{*} \mathrm{a}^{*} \mathrm{~b}^{*}$ and UV Visible spectra, with a measurement time in the region of $2.5 \mathrm{~s}$ per sample (X-Rite Europe $\mathrm{GmbH}$, Regensdorf, Switzerland; Datacolor, Lawrenceville, NJ, USA). The instrument was developed for the printing industry and its application to cultural heritage applications is novel. Samples of approximately 0.05 grams are pressed under 10 tons of force for 120 seconds to obtain a disk approximately $1 \mathrm{~cm}$ in diameter with a flat and uniform surface. Analysis is then performed using EFI Colour Profiler for Printers and ColorPort 1.0.1. software (EFI Colour Profiler for Printers Windows XP 2004 v1.5.9.5). Spectral data was then analysed using single linkage dendrogram routines in MINITAB (Minitab v15.1.30.0; Minitab Inc., State College, PA, USA).

\section{Results}

\section{Microscopy}

When the dust-like particles collected from the rock surface were examined microscopically, a number of components were observed (Figure 3 ). On the basis of their optical appearance, the particles were tentatively identified as quartz (both pure and with inclusions including rhine- stone and haematite), pure haematite, a variety of plant materials, and a variety of pollen species. Whilst Figure 3 is a singular representation of the composition of many of the samples, the microscopy study identified a large variety in particle size and composition, between and within samples. This considerable variation makes conclusions difficult to draw based only on the microscopy study.

\section{X-ray diffraction}

X-ray diffraction analysis identified the presence of quartz in all samples. However, no other mineral components were able to be identified with this method. This was not unexpected, but is valuable as it confirms that the samples being analysed are not contaminated with any ochre material from the surface of the rock. It is important to note that some peaks in the spectra were unable to be identified. This may be due to the presence of low concentrations of components observed with microscopy, such as haematite; however the presence of hematite is unable to be confirmed. In addition to this, XRD analysis is only able to detect components that are crystalline, therefore the possible presence of amorphous or non-crystalline components cannot be discounted. Unfortunately, as only quartz and silicon powder (National Bureau of Standards, now NIST SRM 640a (USA), from the phase reference) were detected, Rietveld quantitative phase analysis was not able to be applied.

\section{Thermal gravimetric analysis}

Thermal gravimetric analysis results are presented in the form of a derivative weight loss curve as this allows observation of the temperature at which the most significant changes take place (Figure 4).

Visual examination of the trends indicated substantial variation between sample types. The spectra can be loosely separated into three groups: i) those showing no mass loss (blue), which are suspected of being salt leached from the rock; ii) those showing three peaks of high intensity at $100,250-275$ and $425-450^{\circ} \mathrm{C}$ (red) are samples collected from the surface of the rock; and iii) those showing 2-3 peaks of medium intensity at 300,400 and $475-500^{\circ} \mathrm{C}$ (green) which are soil and plant samples collected from the caged and surrounding areas.

\section{EFI ES-1000}

The EFI ES-1000 produces a number of characteristic spectroscopic results for each sample analysed, of which the most relevant to this project is the spectral output. Figure 5 displays the first derivative of these spectra, which was chosen because it highlights subtle variations between the spectra of samples that otherwise appear very similar.

Upon inspection of Figure 5, three distinct and separate groups are apparent. The distinction is most noticeable when examining the area between 520 and $620 \mathrm{~nm}$. The group of samples with the greatest intensity in this area (samples 1, 2, 5, 6 and 12) correspond to those samples collected from the rock surface, shown in red. The ground of samples with the lowest intensity in this area (samples 7, 8, 9, 10 and 11) correspond to those samples, both soil and plant material, collected from ground within the caged area and are shown in blue, and the samples with intensities in the middle (samples 3 and 4 ) correspond to those samples collected from the surface of the rock from the area suspected to be contaminated by salt leaching and are shown in green.

\section{Data analysis}

Whilst three distinct groupings of sample types have been shown to be present in the

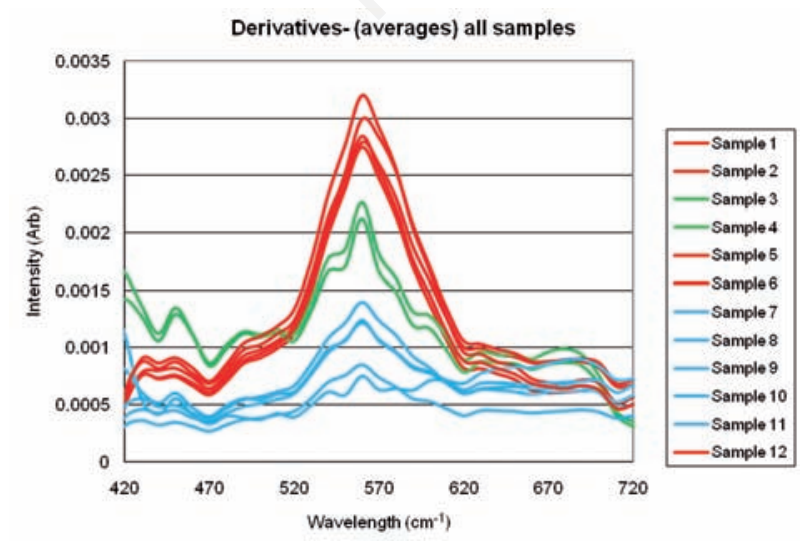

Figure 5. The derivative of the spectra obtained for all samples collected from both the surface of the rock and the ground within the caged area, using the EFI ES-1000 hand-held spectrophotometer.

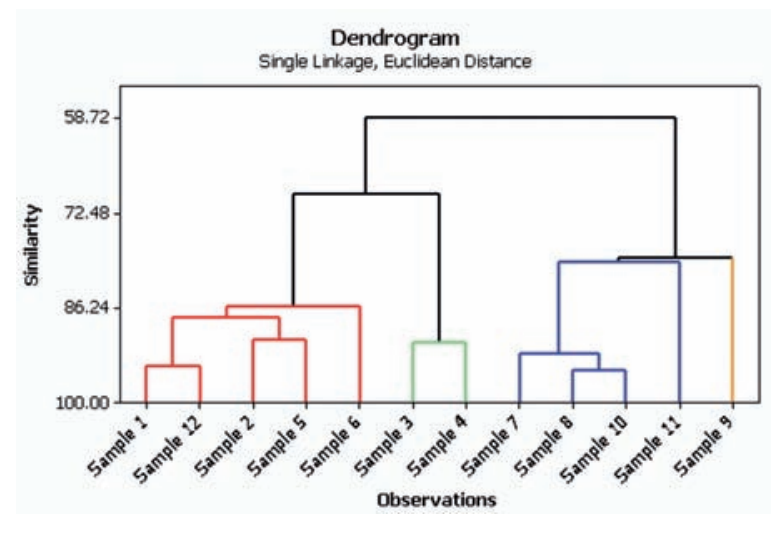

Figure 6. Dendrogram from statistical analysis using the MINITAB software of the derivatives of the spectra obtained for the samples collected (numbering shown in Figure 2) and analysed using the EFI ES-1000. 
results of both TGA and the EFI ES-1000 analyses, interpretation was carried out by visual inspection of the result and as such, may be susceptible to human error. In an attempt to eliminate this, statistical analysis and grouping was undertaken to validate the interpretation.

Statistical analysis in this study was carried out utilising the cluster analysis routines in MINITAB software to produce dendrograms. These are visual illustrations that produce a hierarchical arrangement of sample clusters based on how similar (or dissimilar) objects under analysis (in this case samples) are. Analysis was performed with the spectroscopic results presented in Figure 3, using data in the region of $520-600 \mathrm{~cm}^{-1}$. The method was single linkage, Euclidean distance, and the results of the statistical analysis are shown in Figure 6.

Analysis of the dendrogram reveals four distinct groups of samples, shown in red, green, blue and orange. Further investigation shows that these groups correspond to samples removed from the rock surface, samples removed from the rock surface in an area suspected to be contaminated by salt leaching, samples collected from the ground within the caged area, and plant material, respectfully. These are the distinctions that were visually apparent in Figures 4 and 5.

\section{Discussion}

Results of the microscopy and XRD studies, whilst valuable in confirming a number of similarities between the samples, and in identifying a number of expected components, proved to be of little value statistically and were unable to support or discount the original hypothesis that the substance found on the surface of the rockart was the local soil adhering to the surface of the artwork with the morning dew.

The TGA study, however, proved to be of more value. The peak featured at $100^{\circ} \mathrm{C}$ is unusual because it is a result of the sample drying and water being lost, and as it is only present on those samples collected from the surface of the rock. Its presence supports the hypothesis that the fine particles collected are adhering to the surface of the rock when dew is present.

In addition, TGA analysis was able to separate the samples into three distinct groups: i) those collected from the surface of the rock, ii) those collected from the floor within the protective fence and surrounding area and iii) those suspected of being affected by salt leaching. These results support the hypothesis that the particulate matter adhering to the surface of the rock art is not the same as the material found within the caged area. This is a significant finding for future conservation planning, and supports the hypothesis that the dust-like particles adhering to the rock surface are being carried in on the breeze and are not a result of soil disturbance from within the protective fence area.

The colour analysis results obtained from the EFI ES-1000 also proved to be valuable and visually confirmed the presence of three distinct groups: i) those collected from the surface of the rock, ii) those collected from the floor within the protective fence and surrounding area and iii) those suspected of being effected by salt leaching. Importantly, these are the same three groups as the TGA study showed, and this again supports the hypothesis that the dust-like particles present on the surface of the rock are not from within the protective fence but are in fact from an alternative location.

Statistically, these three groups were confirmed with the production of a dendrogram from MINITAB. Results of this also resulted in further separation with the plant material able to be distinctly separated from the soil samples collected from within the protective fence area.

This statistical analysis supports the hypothesis that the dust-like particles collected from the surface of the rock are not a result of the soil within the caged area being disturbed and adhering to the surface of the rock. The dendrogram shows that these two categories of samples vary by approx $45 \%$ in the region studied. It is also significant to note that statistical analysis was able to further separate the particles adhering to the surface of the rock based on the location of collection, with samples adhering to the south side of the rock able to be statistically separated to those adhering to the north. This is significant as it suggests that the particles are not uniform across the surface, and perhaps are being blown in on the wind from a number of alternate locations with varying wind direction. This requires further investigation.

\section{Conclusions}

Results of microscopy and XRD confirmed the samples contained multiple components, including but not exclusive to quartz and possible pollens. TGA analysis was used and a large water peak was present only in the samples collected from the surface of the rock, supporting the hypothesis that the fine particles collected are adhering to the surface of the rock when dew is present. TGA analysis also confirmed the presence of three distinct groupings within the samples, clearly separating those collected from the surface of the rock from those within the protective fence area, supporting the hypothesis that the particles are travelling on the breeze from an alternate location. The same groups defined in TGA analysis were established with the use of the EFI ES-1000, and further confirmed with statistical analysis. With all the techniques applied showing consistent results, it is concluded that the dust-like particles that are accumulating on the surface of Arkaroo Rock in the Flinders Ranges have not resulted from the removal of plant life and construction of the boardwalk, nor a result of visitors or nature having disturbed the soil from within the caged area. Instead, the particles have travelled in on the breeze from at least one other (unknown) location and adhere to the surface of the rock when dew is present.

\section{References}

Bell IM, Clark RJH, Gibbs PJ, 1997. Raman spectroscopic library of natural and synthetic pigments (pre- [approximate] $1850 \mathrm{AD}$ ). Spectrochim Acta A 53:2159-79.

Bikiaris D, Daniilia S, Sotiropoulou S, Katsimbiri 0, Pavlidou E, Moutsatsou AP, 2000. Ochre-differentiation through microRaman and micro-FTIR spectroscopies: application on wall paintings at Meteora and Mount Athos, Greece. Spectrochim Acta A 56:3-18.

Birt P, 2007. A report for the Department of Environment and Heritage and the Aboriginal Heritage Branch. Aboriginal Affairs and Reconciliation Division, Adelaide.

Elias M, Chartier C, Pr $\leftrightarrow$ evot G, Garay H, Vignaud C, 2006. The colour of ochres explained by their composition. Mater Sci Eng B 127:70-80.

Jercher M, Pring A, Jones PG, Raven MD, 1998. Rietveld X-ray diffraction and X-ray fluorescence analysis of Australian aboriginal ochres. Archaeometry 40:383-401.

Klaassen N, 2007. Flinders ranges research: art in the flinders rangers. South Australia, Adelaide.

Marshall L-JR, Williams JR, Almond MJ, Atkinson SDM, Cook SR, Matthews W, 2005. Analysis of ochres from Clearwell Caves: the role of particle size in determining colour. Spectrochim Acta A 61:233-41.

Morwood M, 1994. Handy household hints for archaeological excavations at rock art sites. Rock Art Res 11:10-2.

Sagona A, 1994. Bruising the red earth. Melbourne University Press, Melbourne.

Watchman AL, Jones R, 1998. Dating rock images in the tropical monsoon region of northern Australia. Aust Aborig Stud 1998:64. 